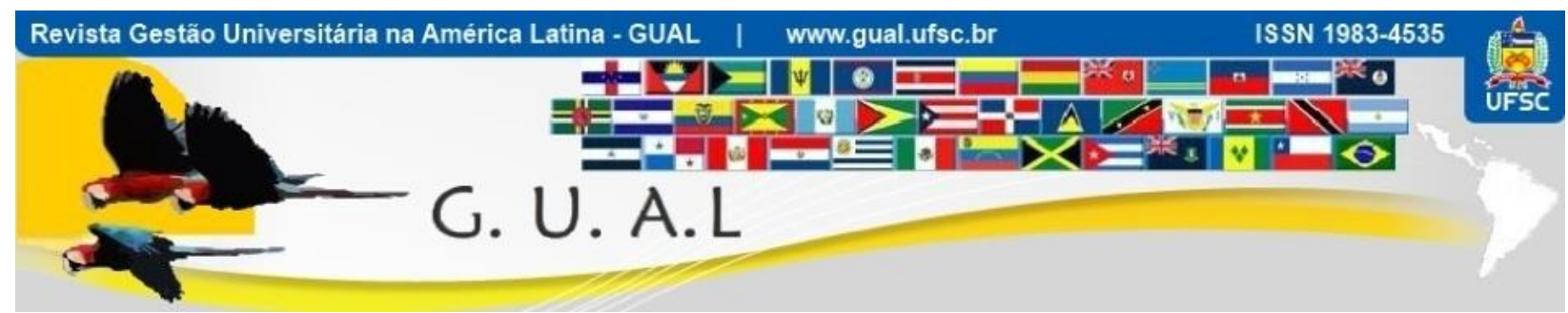

DOI: http://dx.doi.org/1983-4535.2014v7n3p291

\title{
ATRIBUTOS DE QUALIDADE DOS SERVIÇOS PRESTADOS POR UMA IES E OS FATORES QUE IMPACTAM NA SATISFAÇÃO DOS ALUNOS DO CURSO DE GRADUAÇÃO EM ADMINISTRAÇÃO
}

\author{
QUALITY ATTRIBUTES OF SERVICES PROVIDED BY A HEI AND THE \\ FACTORS THAT IMPACT IN THE UNDERGRADUATE STUDENTS OF THE \\ ADMINISTRATION COURSE' SATISFACTION
}

Gabriel Sperandio Milan, Doutor Universidade de Caxias do Sul - UCS gsmilan@ucs.br

Fabiano Larentis, Doutor Universidade de Caxias do Sul - UCS fabiano.larentis@ucs.br

Anderson Corso, Mestre Universidade de Caxias do Sul - UCS acorso@ucs.br

Luciene Eberle, Doutora Universidade de Caxias do Sul - UCS leberle@ucs.br

Fernanda Lazzari, Doutora Universidade de Caxias do Sul - UCS fernandalazzari@hotmail.com

Deonir De Toni, Doutor Universidade de Caxias do Sul - UCS dtoni2@ucs.br

Recebido em 10/março/2013

Aprovado em 02/agosto/2014

Sistema de Avaliação: Double Blind Review

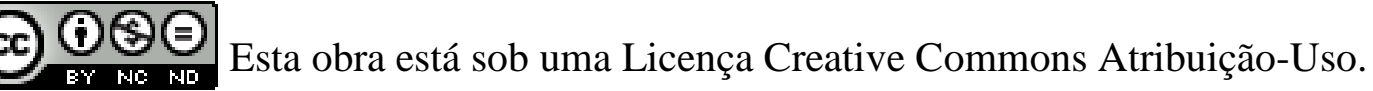




\title{
RESUMO
}

O presente trabalho apresenta um estudo sobre a identificação dos atributos e das dimensões (fatores) da qualidade dos serviços prestados por uma Instituição de Ensino Superior (IES) que impactam na satisfação dos alunos do curso de Administração. A operacionalização da pesquisa se deu, inicialmente, por meio de um estudo qualitativo de caráter exploratório, com aplicação de dezessete entrevistas individuais, com uma abordagem semi-estruturada, mediante a aplicação de um roteiro de questões. As entrevistas foram analisadas a partir de uma análise de conteúdo e geraram atributos (itens ou variáveis observáveis) intrínsecos à qualidade dos serviços prestados pela IES. Com base nos atributos identificados na pesquisa qualitativa, foi elaborado um questionário estruturado, o qual foi aplicado aos alunos do curso de graduação em Administração e resultou em 346 casos válidos. Por meio da análise fatorial, os atributos identificados foram agrupados em seis dimensões de análise ou fatores: corpo docente e currículo, imagem e reputação da IES, valor, limpeza das instalações, atendimento aos alunos e segurança. Além disso, por meio da regressão linear múltipla foi possível explicar $52 \%$ da satisfação geral dos alunos em função de quatro fatores.

Palavras-chave: Qualidade em Serviços. Dimensões da Qualidade em Serviços. Satisfação de Clientes. Serviços Educacionais. Gestão de Serviços Educacionais.

\begin{abstract}
This paper presents a study about how to identify the attributes and services quality dimensions (factors), provided by a Higher Education Institution (HEI) that impact in the students of the Administration course' satisfaction. The operationalization of the research was carried out initially by means an qualitative study with exploratory character, with application of seventeen individual interviews, using a semi-structured approach. A set of questions was applied. The interviews were analyzed using content analysis and ended up generating attributes (items or observable variables) that can be considered intrinsic to the services quality provided by a HEI. Based on attributes identified in the qualitative research, a structured questionnaire was applied for the students of the Administration course and resulted in 346 valid cases. Through factor analysis, the attributes identified were grouped into six analytical dimensions or factors: faculty and curriculum, image and reputation of the HEI, value, cleanliness of facilities, student services and safety. Moreover, by means of multiple linear regression it was possible to explain $52 \%$ of the overall student satisfaction according to four factors.
\end{abstract}

Keywords: Services Quality. Services Quality Dimensions. Customer Satisfaction. Educational Services. Educational Services Management. 


\section{INTRODUÇÃO}

Tendo em vista o acirramento do ambiente concorrencial, as empresas já não podem se considerar detentoras de um determinado market share, visto que os competidores, atuais ou novos entrantes, podem vir de qualquer parte do mercado ou se configurar nos mais variados tipos de organização (O’NEIL; PALMER, 2004). Este contexto de mercado também se aplica aos serviços prestados na área da educação, o que fez com que as Instituições de Ensino Superior (IESs) percebessem a necessidade de buscar diferenciais que atendam às expectativas dos seus clientes (alunos).

As IES, portanto, estão cada vez mais preocupadas em avançar no propósito de se tornarem competitivas na percepção de seus clientes, por meio da valorização dos atributos inerentes ao serviço, principalmente no que diz respeito à qualidade de seus cursos e de sua infraestrutura (SULTAN; WONG 2013). Estes fatores se intensificaram, considerando que, na última década, o ensino superior brasileiro registrou uma expansão acelerada, motivada pela ampliação da abertura de novas IESs e pelo reconhecimento contemporâneo da relação entre qualificação profissional e empregabilidade (LOURENÇO; KNOP, 2011).

Neste sentido, a qualidade na prestação de serviços nas IES tem sido abordada em vários estudos, por diversos autores, no mundo todo, como sendo um fator de consolidação e de alavancagem no mercado (MEYER Jr.; LOPES, 2004). Para que as instituições sejam competitivas e bem-sucedidas em mercados de concorrência acirrada, elas devem fazer um trabalho de excelência na formulação de estratégias e em suas práticas se quiserem ser bemsucedidas em mercados de concorrência acirrada.

Para Kotler e Fox (1994), toda IES deve fazer levantamentos para medir a satisfação dos clientes (alunos, estudantes), pesquisar suas necessidades, desejos e preferências ocultas, a fim de encontrar um modo de melhorar seus serviços. Por conseguinte, uma IES, para responder ao seu mercado, deve se esforçar para sentir, atender e satisfazer às necessidades e aos desejos de seus consumidores e demais públicos.

Com o intuito de realizar um monitoramento constante do nível de satisfação dos alunos, para que se consiga retê-los, fazendo com que se sintam parte integrante da instituição e de seus programas (GREY, 2004), as IES precisam implantar processos de melhoria contínua da qualidade. Estes processos, por sua vez, precisam levar em conta os objetivos da instituição e os anseios de seus alunos, com o intuito de reforçar a possibilidade de recompra 
dos serviços prestados aos alunos egressos e, também, para atrair novos alunos, tornando-a mais competitiva, lucrativa e rentável (MILAN; DE TONI; MAIOLI, 2013).

Frente a isso, esta pesquisa teve como objetivo, inicialmente, identificar os atributos da qualidade dos serviços prestados por uma IES, por meio de uma pesquisa qualitativoexploratória com a coordenação, alunos e professores de um curso de graduação. Na sequência, propôs-se a identificar os fatores ou as dimensões da qualidade destes serviços e, por fim, verificar os fatores que mais impactam na satisfação geral dos alunos em relação aos serviços educacionais prestados.

O esforço de pesquisa acerca desta temática se justifica pelo fato de que atitudes positivas na prestação de serviço levam ao aumento considerável da retenção de clientes. A desistência ou a perda de um cliente (aluno) diminui a receita da IES e também pode gerar o enfraquecimento de sua imagem entre os candidatos potenciais. Por sua vez, a satisfação do cliente (aluno) e a percepção de qualidade superior incentivam comportamentos favoráveis em relação à prestação do serviço. Nesta mesma linha de raciocínio, Jongbloed (2003) menciona o crescimento das práticas mercadológicas no ensino superior como um fator que pode contribuir no processo da escolha por uma IES, seja pela inovação seja pela qualidade apresentada ou pela variedade dos serviços oferecidos, contribuindo para uma especial atenção no momento da decisão do aluno.

Segundo Mainardes, Domingues e Deschamps (2009), o Brasil, no ambiente das IESs, tem acompanhado a tendência competitiva através do aumento das habilidades em atrair, satisfazer e reter seus clientes, reforçando aspectos atinentes à qualidade dos serviços prestados. Dessa forma, estabelecer uma relação duradoura com os alunos ajuda na sustentabilidade e no fortalecimento da imagem institucional (ALVES; RAPOSO, 2007).

\section{FUNDAMENTAÇÃO TEÓRICA}

\subsection{ENTENDIMENTO SOBRE A QUALIDADE EM SERVIÇOS EDUCACIONAIS}

Conforme Ostrom e Iacobucci (1995), os serviços podem ser divididos em serviços experienciais e serviços de crença. Nos serviços experienciais, os clientes, após algumas interações com a empresa são capazes de realizar avaliações sobre o desempenho do serviço prestado, enquanto nos serviços de crença há uma maior dificuldade do cliente conseguir realizar uma avaliação, mesmo após recorrentes interações com o provedor de serviço. Neste caso estão os serviços com maiores graus de especialização, que, muitas vezes, estão além da 
compreensão do cliente devido à grande especificidade do que é oferecido pela empresa (serviços médicos, por exemplo).

Desse modo, alguns serviços são mais difíceis de serem julgados do que outros e a excelência em sua execução, dia após dia, é fundamental na construção da confiança do cliente em relação ao provedor de serviços (BERRY, 2001). Nos serviços educacionais, verifica-se que uma educação de qualidade não pode ser construída por sistemas educacionais constituídos por estruturas burocráticas, lentas e centralizadoras e que não permitem agilidade na solução de problemas. Neste contexto, a gestão é um componente decisivo para a eficácia educacional e vem comprovar que as IESs bem dirigidas, estruturadas e focadas no mercado são mais eficientes e eficazes (XAVIER, 1996).

Lovelock (1983) destaca que o serviço educacional se classifica como um serviço de entrega contínua, sendo dirigido à mente das pessoas e com um alto contato pessoal, formado pela parceria entre a organização de serviço e o cliente (aluno). Por sua vez, Morales e Calderón (1999) afirmam que as instituições de ensino, por definição, são fornecedoras de serviços em todas as dimensões e que não devem se resumir apenas à atividade de ensino e aprendizagem. Segundo Jackson, Helms e Ahmadi (2011) o aluno, no momento em que entra em contato com a instituição, começa a receber estímulos que são processados, formando, assim, sua avaliação pessoal da qualidade global do serviço educacional.

Na perspectiva de implementação de novos padrões de gestão, as IES devem priorizar a implementação de sistemas de monitoramento e acompanhamento sistemáticos da qualidade e do desempenho escolar. Neste contexto, Xavier (1996) defende as seis dimensões da qualidade em educação propostas, que devem auxiliar aos gestores educacionais no desenvolvimento de habilidades gerenciais, centradas na qualidade, para auxiliar no momento da tomada de decisões, que são: (i) qualidade política e formal da educação, constituindo o aspecto político-pedagógico da educação; (ii) custo para se obter a educação, considerando os aspectos de quantidade certa de educação, no prazo e local adequados; (iii) moral da equipe envolvida que faz o processo de contato entre aluno e instituição; (iv) moral dos profissionais de educação; (v) segurança; e (vi) ética, que emerge como a última dimensão, no que diz respeito ao comportamento dos atores inseridos no processo educacional.

Os debates sobre a avaliação e a qualidade no ensino superior vêm ganhando espaço na literatura e na mídia, em decorrência das ações governamentais que visam o controle da qualidade do ensino prestado. Porém, é importante atentar para o fato que avaliação e 
mensuração da qualidade em serviços têm de ser realizada de forma relativa, devido à intangibilidade e a natureza abstrata dos serviços (LOPES; LEITE; LEITE, 2007).

Além disso, a expansão do mercado de IES acabou por afetar o nível de ensino. Consoante isso, os gestores de IESs se deparam com o desafio de identificar e implementar o método mais adequado para aferir a qualidade de seus serviços (O’NEIL; PALMER, 2004), especialmente considerando a perspectiva do cliente (aluno), com base em um melhor conhecimento de suas necessidades, desejos, potencialidades e demandas (JACKSON; HELMS; AHMADI, 2011). Uma abordagem com este direcionamento na gestão da qualidade tende a convergir para uma melhora na imagem e na reputação da instituição, bem como para a satisfação e a retenção dos alunos em relação aos serviços prestados pela IES (BERGAMO; GIULIANI; GALLI, 2011).

Sendo assim, no contexto educacional, a qualidade em serviços é entendida como um sistema em que os clientes (alunos) e os fornecedores (IESs) devem estar envolvidos para a satisfação de necessidades e desejos, visando a uma continuidade dos relacionamentos (JACKSON; HELMS; AHMADI, 2011; MILAN; DE TONI; MAIOLI, 2013). Isso ocorre porque importantes fontes de vantagem competitiva sustentáveis são fomentadas pelos elevados níveis de qualidade da IES, o que possibilita a ampliação dos laços de retenção ou de lealdade nos relacionamento com os clientes (REICHHELD; MARKEY Jr.; HOPTON, 2000; BERGAMO; GIULIANI; GALLI, 2011; SULTAN; WONG, 2013).

\subsection{A RELAÇÃO ENTRE A QUALIDADE DOS SERVIÇOS E A SATISFAÇÃO DE CLIENTES}

Observa-se que a qualidade em serviços é uma avaliação focada e reflete na percepção dos clientes sobre fatores específicos dos serviços (confiabilidade, responsividade, segurança, empatia e tangibilidade). Em decorrência disso, o cliente poderá avaliar a qualidade de um provedor de serviços através da confirmação de algumas ou de todas, as dimensões do serviço que foi realizado (PARASURAMAN; ZEITHAML; BERRY, 1985; 1988). Além disso, alguns fatores pessoais também podem influenciar as percepções da qualidade de um produto ou de um serviço, incluindo o estado emocional dos clientes. Portanto, a satisfação dos clientes poderá sofrer influências pelas contrapartidas emocionais dos clientes, percebendo-se as causas para o sucesso ou o fracasso de um provedor de serviços e as suas percepções de ganhos (ZEITHAML; BITNER, 2003). 
Sendo assim, uma organização, no caso do estudo, uma IES, precisa obter sua formação e programas de desenvolvimento alinhados com suas estratégias e metas, para que, desta forma, possa atender às expectativas de seus clientes e, assim, satisfazê-los. Portanto, se a qualidade não tiver destaque na prestação de serviço há um risco maior de perder clientes e também perder vantagens competitivas (REED; VAKOLA, 2006). Seguindo este raciocínio, Elliot e Shin (2002) destacam que no ensino superior a satisfação do aluno tem impacto positivo na captação de recursos e na motivação dos mesmos, pois a satisfação do cliente é mais eficiente e, ao mesmo tempo, a maneira menos custosa de se comunicar e manter os clientes dentro de uma IES (KANJI, 2007).

Vale destacar que a discussão acerca da relação entre a qualidade percebida e a satisfação de clientes é algo bastante presente na literatura. No entanto, Dabholkar (1995) postula a existência de relativo consenso entre os autores, que afirmam que a qualidade consiste em um construto mais simples, abrangendo a dimensão cognitiva, enquanto a satisfação trata-se de um conceito mais complexo, que abrange componentes cognitivos e afetivos. Inclusive, Zeithaml (2000) comenta que a qualidade do serviço é um componente central das percepções do cliente, promovendo uma satisfação quando o serviço ofertado apresentar uma qualidade percebida igual ou superior ao esperado, e insatisfação quando essa qualidade estiver aquém.

Laroche et al. (2004) comentam, ainda, que além da satisfação, a qualidade percebida também está associada às preferências e às expectativas dos clientes. Porém, conforme Lovelock e Wright (2001), a qualidade do serviço e a satisfação do cliente não são exatamente a mesma coisa. Segundo os autores, enquanto a qualidade se configura como uma avaliação global a respeito do serviço, a partir de experiências passadas e de outros aspectos, a satisfação corresponde a uma reação emocional ao desempenho específico do serviço.

Ainda no que tange às diferenças entre os conceitos de qualidade e satisfação, Anderson, Fornell e Lehmann (1994) afirmam que o cliente precisa da experiência, ou seja, de evidências para determinar seu grau de satisfação em relação ao serviço, ao passo que não necessita dela para determinar sua qualidade.

Zeithaml e Bitner (2003), por sua vez, destacam que a qualidade do serviço é um dos componentes da satisfação de clientes, uma vez que a satisfação é influenciada pela qualidade dos serviços, pelo preço e pelas percepções a respeito dos fatores situacionais e pessoais. Nesta direção, Pantouvakis e Lymperopoulus (2008) entendem que a satisfação pode ser vista 
como um resultado comparativo entre a qualidade percebida do serviço pelos clientes e suas expectativas anteriores.

É importante salientar que, conforme Oliver (2010), a qualidade percebida atua como formadora da satisfação em relação aos serviços prestados. Sendo assim, o consumidor realiza a avaliação da qualidade percebida e esta se torna um antecedente da satisfação com a prestação do serviço (OLIVER, 2010). Fornell (1992) ainda aponta para o fato de que, a partir da experiência acumulada que o consumidor possuir com a empresa prestadora de serviços, ocorrerá a formação da satisfação global, que é o resultado da avaliação de todas as interações que o cliente realizou com a empresa. Pode-se dizer, então, que qualidade deve ser entendida como a capacidade intrínseca dos produtos e serviços de prover a satisfação de seus consumidores, a partir da conformidade das especificações do cliente, pois o que conta é o que o cliente percebe como qualidade (Milan; Brentano; De Toni, 2008).

\section{MÉTODO DE PESQUISA}

A pesquisa foi realizada em duas fases. Uma fase qualitativo-exploratória (DENZIN; LINCOLN, 2011), com a implementação de entrevistas individuais (KING; HORROCKS, 2010; GUBRIUM et al., 2012), com uma abordagem semiestruturada mediante a aplicação de um roteiro básico de questões (RIBEIRO; MILAN, 2004). E uma fase quantitativo-descritiva (REMLER; VAN RYZIN, 2011), operacionalizada por meio de uma survey (FINK, 2012), na qual foi aplicado um questionário estruturado junto a estudantes do curso de Administração de uma Instituição de Ensino Superior (IES) localizada no Estado do Rio Grande do Sul (RS).

\subsection{COLETA DOS DADOS}

O instrumento utilizado para coleta de dados compreendeu 53 atributos (itens ou variáveis observáveis) intrínsecos à qualidade dos serviços prestados pela IES, os quais são provenientes de 17 entrevistas individuais realizadas junto ao Coordenador de Curso, professores, alunos e funcionários, cujos dados foram tratados por meio de análise de conteúdo (KRIPPENDORFF, 2012; MILES; HUBERMAN; SALDAÑA, 2013), bem como da revisão da literatura (FERREIRA, 2005; EBERLE; MILAN; LAZZARI; 2010; MILAN; DE TONI; MAIOLI, 2013). A operacionalização dos atributos contemplados se deu por meio de uma escala intervalar de sete pontos do tipo Likert (HAIR Jr. et al., 2010). Esta escala continha, em seus extremos, (1) Totalmente Insatisfeito e (7) Totalmente Satisfeito, exigindo 
que os entrevistados apontassem, em sua percepção, um grau de satisfação no que se referia a cada afirmação proposta, facilitando o entendimento dos respondentes e o tratamento posterior das respostas (ou dados coletados) (FINK, 2012).

Após a validação de conteúdo do questionário (MALHOTRA, 2006) por três experts da área de marketing, que resultou na identificação dos atributos da pesquisa, foi aplicado um pré-teste junto a dez alunos do curso. A aplicação dos questionários ocorreu em diversas turmas do curso de Administração, pelo método de autopreenchimento (HAIR Jr. et al., 2010) dos alunos. No momento da aplicação dos questionários foi dada uma breve explicação do instrumento de dados com o objetivo de reforçar a importância do estudo. Foram coletados dados relativos a 346 alunos, sendo 52,5\% do gênero (sexo) masculino (182 alunos) e 47,5\% do feminino (164 alunas), de acordo com o estabelecido previamente, para que se mantivesse a proporcionalidade existente no curso no que se refere a este critério.

\subsection{ANÁLISE DOS DADOS}

Após a finalização do processo de coleta, passou-se para a depuração do banco de dados, afim de garantir que eventuais inconsistências nos dados não prejudicassem as análises (TABACHNICK; FIDELL, 2012). Não foram identificados padrões de ausência de resposta (missings) nas questões, conforme recomendado pela literatura (DAVEY; SAVLA, 2009; ENDERS, 2010). Entretanto, oito variáveis que apresentaram mais de $10 \%$ de dados faltantes (HAIR Jr. et al., 2009) foram retiradas, reduzindo o conjunto de atributos relacionados à qualidade dos serviços prestados pela IES a 45 itens. Observadas as características das nãorespostas (baixa frequência e aleatoriedade), optou-se pela substituição dos valores faltantes pela média (HAIR Jr. et al., 2009).

Para a análise dos dados atípicos (outliers), optou-se pelo critério multivariado. Um outlier multivariado possui valores extremos em mais de uma variável ou possui uma configuração de respostas incomum (KLINE, 2011). A distância D2 de Mahalanobis mede a distância de cada caso em relação ao centróide, para uma distribuição com determinada covariância. A distribuição da medida D2 é o qui-quadrado com graus de liberdade igual ao número de variáveis. São considerados outliers aqueles casos cujas probabilidades associadas ao valor de D2 forem inferiores a 0,001. Foi identificado um outlier neste conjunto de dados.

Além disso, a realização de análises multivariadas exige que algumas suposições estejam satisfeitas. As principais são a normalidade, a linearidade e a multicolinearidade 
(MALHOTRA, 2006). Uma das formas de analisar a normalidade é a partir dos valores de assimetria e de curtose (HAIR Jr. et al., 2009). A linearidade é outra suposição relevante, pois a maioria das técnicas pressupõe relações lineares entre as variáveis, e pode ser verificada através do estudo dos diagramas de dispersão dos dados (HAIR Jr. et al., 2009). Já a multicolinearidade é observada quando uma variável pode ser explicada pelas outras variáveis da análise (HAIR et at., 2009). Na prática, a multicolinearidade pode ser observada quando as variáveis têm alta correlação entre si, por meio do estudo da matriz de correlações. Hair Jr. et al. (2009) e Kline (2011) sugerem valores aceitáveis até 0,90 e 0,85, respectivamente. As análises de normalidade (univariada), linearidade e multicolinearidade indicaram que os dados estão dentro de faixas adequadas e poderiam ser utilizados nas análises.

Em seguida, foram realizadas análises estatísticas descritivas (cálculo de média e desvio-padrão), análise fatorial e análise de regressão múltipla, a partir das médias dos fatores resultantes como variáveis independentes e da satisfação geral dos alunos como variável dependente (MALHOTRA, 2006).

\section{ANÁLISE DOS RESULTADOS}

\subsection{CARACTERIZAÇÃO DA AMOSTRA}

A amostra compreendeu 346 casos válidos, mais especificamente 182 alunos (52,5\%) e 164 alunas (47,5\%). Estes alunos têm uma média de 26,6 anos de idade (desvio padrão de 5,5 anos) e mediana de 25 anos. 3,2\% deles estão no início do curso (até o $3^{\circ}$ semestre), 40\% no meio do curso (do $4^{\circ}$ ao $7^{\circ}$ semestre) e $56,8 \%$ ao seu final (do $8^{\circ}$ ao $10^{\circ}$ semestre). A renda familiar destes alunos é distribuída da seguinte forma: 18,8\% disseram que a renda familiar é de até $\mathrm{R} \$ 1.000,00,24,3 \%$ entre $\mathrm{R} \$ 1.001,00$ a $1.500,00,38,8 \%$ entre $\mathrm{R} \$ 1.501,00$ a $\mathrm{R} \$ 3.000,00$ e $17,9 \%$ de mais de $\mathrm{R} \$ 3.000,00$.

\subsection{NÍVEIS DE SATISFAÇÃO EM RELAÇÃO AOS ATRIBUTOS DE QUALIDADE DOS SERVIÇOS PRESTADOS}

Como pode ser verificado na Tabela 1 , as médias resultantes da satisfação dos alunos no que diz respeito aos atributos de qualidade dos serviços prestados pela IES variaram de 2,41 (mensalidade do curso) a 5,61 (atendimento na biblioteca). A satisfação geral dos alunos ficou em 4,39. Ou seja, acima da satisfação relativa a 34 atributos (itens) avaliados e abaixo somente de 11 deles. As melhores médias estão associadas à qualidade do corpo docente, 


\section{ATRIBUTOS DE QUALIDADE DOS SERVICOS PRESTADOS POR UMA IES E OS FATORES QUE IMPACTAM NA SATISFAÇÃO DOS ALUNOS DO CURSO DE GRADUAÇÃO EM ADMINISTRAÇÃO DOI: http://dx.doi.org/1983-4535.2014v7n3p291}

biblioteca, atendimento e conveniência. As piores médias estão associadas aos preços e ao valor para o cliente, alguns aspectos estruturais e atinentes a serviços complementares.

Tabela 1 Satisfação dos alunos em relação aos atributos avaliados e sua satisfação geral

\begin{tabular}{|c|c|c|c|}
\hline N. & Atributos de Qualidade dos Serviços Prestados pela IES & $\begin{array}{l}\text { Satisfação dos Alunos } \\
\text { (Média) }\end{array}$ & $\begin{array}{l}\text { Desvio } \\
\text { Padrão }\end{array}$ \\
\hline 1 & Atendimento na biblioteca & 5,61 & 1,24 \\
\hline 2 & Coordenação do curso & 5,56 & 1,29 \\
\hline 3 & Limpeza das salas de aula & 5,51 & 1,26 \\
\hline 4 & Acervo biblioteca & 5,46 & 1,16 \\
\hline 5 & Limpeza dos corredores & 5,43 & 1,20 \\
\hline 6 & Atendimento na secretaria & 5,40 & 1,23 \\
\hline 7 & Avaliação on line & 5,23 & 1,29 \\
\hline 8 & Localização da IES & 5,21 & 1,26 \\
\hline 9 & Serviços disponíveis via biblioteca virtual & 5,21 & 1,25 \\
\hline 10 & Disponibilidade de diversos núcleos & 5,21 & 1,22 \\
\hline 11 & Titulação do corpo docente & 5,19 & 1,17 \\
\hline 12 & Atendimento no protocolo & 5,18 & 1,32 \\
\hline 13 & Experiência profissional do corpo docente & 5,11 & 1,19 \\
\hline 14 & Qualificação do corpo docente & 5,10 & 1,23 \\
\hline 15 & Cumprimento do plano de ensino da disciplina & 5,02 & 1,19 \\
\hline 16 & Imagem da IES & 4,99 & 1,27 \\
\hline 17 & Flexibilidade de turnos e/ou horários & 4,99 & 1,40 \\
\hline 18 & Infraestrutura de IES & 4,99 & 1,27 \\
\hline 19 & Aplicabilidade dos conteúdos & 4,96 & 1,05 \\
\hline 20 & Conveniência dos serviços complementares & 4,90 & 1,60 \\
\hline 21 & Critérios de avaliação das disciplinas & 4,88 & 1,24 \\
\hline 22 & Organização/divisão dos blocos & 4,88 & 1,18 \\
\hline 23 & Reputação da IES & 4,87 & 1,22 \\
\hline 24 & Disponibilidade de estágios, monitorias e intercâmbios & 4,84 & 1,24 \\
\hline 25 & Flexibilidade de disciplinas & 4,84 & 1,24 \\
\hline 26 & Confiança na IES & 4,82 & 1,31 \\
\hline 27 & Local de integração para os alunos & 4,79 & 1,40 \\
\hline 28 & Grade curricular & 4,78 & 1,45 \\
\hline 29 & Segurança nas instalações da IES & 4,77 & 1,45 \\
\hline 30 & Limpeza dos banheiros & 4,72 & 1,62 \\
\hline 31 & Ações de comunicação & 4,72 & 1,20 \\
\hline 32 & Integração entre as disciplinas do curso & 4,59 & 1,26 \\
\hline 33 & Comprometimento da IES com os alunos & 4,58 & 1,31 \\
\hline 34 & Relevância das disciplinas & 4,43 & 1,20 \\
\hline 35 & Laboratórios de apoio & 4,37 & 1,54 \\
\hline 36 & Serviços de reprografia & 4,23 & 1,41 \\
\hline 37 & Possibilidade de participar de atividades relacionadas a pesquisas & 4,21 & 1,34 \\
\hline 38 & Estruturas das salas de aula & 4,20 & 1,66 \\
\hline
\end{tabular}




\begin{tabular}{c|l|l|l}
\hline 39 & Segurança nos estacionamentos da IES & 4,15 & 1,62 \\
\hline 40 & Número de alunos por turma & 3,73 & 1,61 \\
\hline 41 & Cota de reprografia por disciplina & 3,36 & 1,90 \\
\hline 42 & Relação custo versus benefícios & 3,35 & 1,60 \\
\hline 43 & Valor da mensalidade da IES em comparação a outras & 3,29 & 1,45 \\
\hline 44 & Área de estacionamento & 3,19 & 1,83 \\
\hline 45 & Mensalidade do curso & 2,41 & 1,55 \\
\hline \multicolumn{2}{l|}{ Satisfação Geral dos Alunos } & $\mathbf{4 , 3 9}$ & $\mathbf{1 , 0 9}$ \\
\hline
\end{tabular}

Fonte: Dados provenientes da pesquisa.

Obs.: Escala utilizada: (1) Totalmente Satisfeito a (7) Totalmente Insatisfeito.

\subsection{ANÁLISE FATORIAL}

Procedeu-se a Análise Fatorial Exploratória (AFE), com base no método ortogonal de rotação fatores, denominada de rotação Varimax, a qual tem por finalidade uma melhor interpretação e análise destes fatores. É oportuno comentar que foi adotado como critério para a definição do número de fatores aqueles com autovalor superior a um e, para retirada das variáveis, as que apresentassem uma carga fatorial inferior a 0,5 e comunalidade igualmente inferior a 0,5 (JOHNSON; WICKERN, 2007; MULAIK, 2010). Em função disso, foram excluídas 14 variáveis (atributos) em seis rodadas de análise. Partiu-se de onze fatores na primeira rodada de análise até oito fatores na sétima rodada. Vale destacar que na sétima rodada, apesar de todas as variáveis apresentarem cargas fatoriais superiores a 0,5, havia um fator com apenas uma variável (estrutura das salas). Pensando-se em parcimônia do número de fatores, foi executada uma análise com sete fatores, com a eliminação de uma variável com carga inferior a 0,5 .

Com sete fatores, resultando em 28 variáveis (atributos), procederam-se os cálculos de suas confiabilidades, a partir do Alfa de Cronbach. Seis fatores apresentaram alfas superiores a 0,7 , enquanto um deles inferior a 0,5 , englobando as variáveis "critérios de avaliação das disciplinas", "disponibilidade de diversos núcleos" e "cota de reprografia por disciplina". Em função disso, optou-se por implementar uma análise fatorial fixando seis fatores. Em decorrência disso, foram eliminadas mais 4 variáveis com carga fatorial inferior a 0,5 , e com a composição dos fatores muito semelhante à análise anterior, daqueles com alfa superior a 0,7.

A análise fatorial com seis fatores, com 24 variáveis (atributos) resultantes, obteve um KMO de 0,89 e teste de esfericidade de Bartlett com significância inferior a 0,001, em níveis dentro dos parâmetros estabelecidos para a adequação do uso da análise fatorial para os dados do estudo (JOHNSON; WICKERN, 2007). A variância explicada resultou em 65,1\%, assim 
como os Alfas de Cronbach de cada fator, superiores a 0,7, o que é convergente com patamares recomendados (HAIR Jr. et al., 2009; MALHOTRA, 2006). Na Tabela 2 são apresentados os fatores Identificados, suas respectivas variáveis e cargas fatoriais, Alfas de Cronbach e variâncias explicadas.

Tabela 2 Fatores identificados e seus respectivos atributos de qualidade dos serviços

\begin{tabular}{|c|c|c|c|c|c|c|}
\hline $\begin{array}{c}\text { Fatores } \\
\text { Identificados }\end{array}$ & $\begin{array}{c}\text { Variáveis } \\
\text { (Atributos de Qualidade dos Serviços) } \\
\end{array}$ & $\mathbf{C F}$ & $\mathrm{AC}$ & $\begin{array}{r}\text { VET } \\
65,1 \% \\
\end{array}$ & $\mathbf{M}$ & DP \\
\hline \multirow{6}{*}{$\begin{array}{c}\text { F1 - Corpo } \\
\text { Docente e } \\
\text { Currículo }\end{array}$} & Qualificação do corpo docente & 0,756 & \multirow{6}{*}{0,835} & \multirow{6}{*}{$34,1 \%$} & \multirow{6}{*}{5,00} & \multirow{6}{*}{0,92} \\
\hline & Experiência profissional do corpo docente & 0,706 & & & & \\
\hline & Grade curricular & 0,677 & & & & \\
\hline & Titulação do corpo docente & 0,651 & & & & \\
\hline & Cumprimento do plano de ensino da disciplina & 0,611 & & & & \\
\hline & Flexibilidade de disciplinas & 0,610 & & & & \\
\hline \multirow{5}{*}{$\begin{array}{c}\text { F2 - } \\
\text { Imagem e } \\
\text { Reputação }\end{array}$} & Confiança na IES & 0,784 & \multirow{5}{*}{0,862} & \multirow{5}{*}{$8,2 \%$} & \multirow{5}{*}{4,86} & \multirow{5}{*}{1,02} \\
\hline & Comprometimento da IES com os alunos & 0,737 & & & & \\
\hline & Imagem da IES & 0,696 & & & & \\
\hline & Infraestrutura de IES & 0,669 & & & & \\
\hline & Reputação da IES & 0,617 & & & & \\
\hline \multirow{4}{*}{ F3 - Valor } & Mensalidade do curso & 0,793 & \multirow{4}{*}{0,737} & \multirow{4}{*}{$6,6 \%$} & \multirow{4}{*}{3,11} & \multirow{4}{*}{1,22} \\
\hline & $\begin{array}{l}\text { Valor da mensalidade da IES em comparação a } \\
\text { outras }\end{array}$ & 0,721 & & & & \\
\hline & Relação custo versus benefícios & 0,717 & & & & \\
\hline & Cota de reprografia por disciplina & 0,600 & & & & \\
\hline \multirow{3}{*}{$\begin{array}{l}\text { F4 - } \\
\text { Limpeza }\end{array}$} & Limpeza dos corredores & 0,839 & \multirow{3}{*}{0,861} & \multirow{3}{*}{$6,1 \%$} & \multirow{3}{*}{5,22} & \multirow{3}{*}{1,21} \\
\hline & Limpeza das salas de aula & 0,836 & & & & \\
\hline & Limpeza dos banheiros & 0,832 & & & & \\
\hline \multirow{4}{*}{$\begin{array}{c}\text { F5 - } \\
\text { Atendimento } \\
\text { aos Alunos }\end{array}$} & Atendimento na secretaria & 0,761 & \multirow{4}{*}{0,722} & \multirow{4}{*}{$5,3 \%$} & \multirow{4}{*}{5,44} & \multirow{4}{*}{0,94} \\
\hline & Atendimento no protocolo & 0,683 & & & & \\
\hline & Atendimento na biblioteca & 0,670 & & & & \\
\hline & Coordenação do curso & 0,578 & & & & \\
\hline \multirow{2}{*}{$\begin{array}{l}\text { F6 - } \\
\text { Segurança }\end{array}$} & Segurança nas instalações da IES & 0,781 & \multirow{2}{*}{0,799} & \multirow{2}{*}{$4,7 \%$} & \multirow{2}{*}{4,48} & \multirow{2}{*}{1,39} \\
\hline & Segurança nos estacionamentos da IES & 0,771 & & & & \\
\hline
\end{tabular}

Fonte: Dados provenientes da pesquisa.

Legenda: CF: Carga Fatorial; AC: Alfas de Cronbach; VET: Variância Explicada Total; M: Média; DP: Desvio Padrão.

Conforme mostra a Tabela 2, os seis fatores (ou dimensões da qualidade) estão associados à execução do ensino, mais especificamente ao Corpo Docente e Currículo do curso (F1), à Imagem e Reputação da IES (F2), à relação de Valor (F3) e ao Atendimento aos Alunos (F5), à Limpeza das instalações físicas (F4) e à Segurança (F6). Com exceção dos fatores Limpeza (F4) e Segurança (F6), com seus respectivos atributos (ou variáveis), outros elementos mais associados a aspectos estruturais e tangíveis não permaneceram na 
análise final. Isto pode ter ocorrido devido à natureza do curso investigado (Administração), um curso de natureza predominantemente teórica.

Cabe ressaltar que um dos fatores, Valor (F3), apresentou uma média geral inferior a 4 (ponto médio da escala, a qual varia de 1 a 7). Outros dois fatores apresentaram médias entre 4 e 5 (Segurança - F6 e Imagem e reputação - F2). Por outro lado, o restante dos fatores apresentou média superior a 5 (Corpo Docente e Currículo - F1, Limpeza - F4 e Atendimento aos Alunos - F5).

\subsection{CORRELAÇÕES E ANÁLISE DE REGRESSÃO}

Em acréscimo, foram calculadas as correlações entre os fatores e o grau de satisfação geral dos alunos, implementando-se uma análise de regressão (MEYERS; GAMST; GUARINO, 2012). Quanto à análise das correlações, de acordo com a Tabela 3, para fins comparativos, foram identificadas nove correlações superiores a 0,45 , praticamente todas elas relacionadas ao Corpo Docente e Currículo (F1), à Imagem e Reputação da IES (F2), ao Atendimento aos Alunos (F5) e ao Valor (F3), ou seja fatores associados à execução do ensino, ao atendimento aos alunos (serviços prestados), ao valor deste atendimento e destes serviços para o aluno, e à imagem e reputação da IES, aspectos de cunho mais intangível. Portanto, a satisfação geral, variável de natureza dependente, apresentou as maiores correlações com tais fatores.

Tabela 3 Matriz de correlação (Pearson)

\begin{tabular}{l|c|c|c|c|c|c|c}
\hline \multicolumn{1}{c|}{ Fatores } & F1 & F2 & F3 & F4 & F5 & F6 & Satisfação Geral \\
\hline F1 - Corpo Docente e Currículo & 1 & & & & & & \\
\hline F2 - Imagem e Reputação & $\mathbf{0 , 6 0 7}$ & 1 & & & & & \\
\hline F3 - Valor & 0,372 & $\mathbf{0 , 4 5 1}$ & 1 & & & & \\
\hline F4 - Limpeza & 0,444 & 0,385 & 0,231 & 1 & & & \\
\hline F5 - Atendimento aos Alunos & $\mathbf{0 , 5 0 3}$ & $\mathbf{0 , 4 5 7}$ & 0,321 & 0,394 & 1 & & \\
\hline F6 - Segurança & 0,409 & $\mathbf{0 , 5 0 7}$ & 0,382 & 0,371 & 0,342 & 1 & \\
\hline Satisfação Geral & $\mathbf{0 , 5 3 1}$ & $\mathbf{0 , 6 3 6}$ & $\mathbf{0 , 5 3 6}$ & 0,386 & $\mathbf{0 , 4 9 7}$ & 0,444 & 1 \\
\hline
\end{tabular}

Fonte: Dados provenientes da pesquisa.

A análise de regressão teve como variáveis independentes os seis fatores e como variável dependente a satisfação geral. Para tanto, procedeu-se a verificação da correlação item-total máxima de 0,8 para cada variável, garantindo que não haja multicolinearidade (KLINE, 2011). Adicionalmente, dentro das estatísticas de colinearidade, se avaliou, para 
cada variável independente, se os valores de tolerância de cada item eram maiores que o valor resultante de 1 menos o $\mathrm{R}^{2}$ de cada modelo (LEECH; BARRETT; MORGAN, 2005). Em todas as análises os valores das tolerâncias foram superiores aos de (1- $\left.\mathrm{R}^{2}\right)$, não apresentando problema de multicolinearidade.

A partir do método stepwise, o qual considera apenas as variáveis independentes estatisticamente significantes (ao nível de 0,05) (HAIR Jr. et al., 2009), o modelo de regressão, com um coeficiente de determinação $\left(R^{2}\right)$ de 0,52 , repercutindo em um bom poder de explicação, consta de quatro fatores, em ordem decrescente de coeficientes padronizados (betas): Imagem e Reputação (F2), Valor (F3), Atendimento aos Alunos (F5) e Corpo Docente e Currículo (F1). Ou seja, estes são os fatores de maior impacto na satisfação geral dos alunos do curso de Administração da IES pesquisada. Salienta-se, também, que dois destes fatores, Imagem e Reputação (F2) e Valor (F3), obtiveram betas superiores a 0,25. Os resultados da análise de regressão são apresentados na Tabela 4.

Tabela 4 Resultados da análise de regressão

\begin{tabular}{l|c|c|c|c}
\hline \multicolumn{1}{c|}{ Fatores } & $\begin{array}{c}\text { Coeficientes Não } \\
\text { Padronizados } \\
\text { (constante -0,095) }\end{array}$ & $\begin{array}{c}\text { Coeficientes } \\
\text { Padronizados (Betas) }\end{array}$ & Valor t & Sig. \\
\hline F2 - Imagem e Reputação & 0,377 & 0,354 & 7,083 & 0,000 \\
\hline F3 - Valor & 0,243 & 0,271 & 6,404 & 0,000 \\
\hline F5 - Atendimento aos Alunos & 0,218 & 0,187 & 4,208 & 0,000 \\
\hline F1 - Corpo Docente e Currículo & 0,143 & 0,121 & 2,437 & 0,015 \\
\hline
\end{tabular}

Fonte: Dados provenientes da pesquisa.

Desta maneira, para fins preditivos, a Satisfação Geral dos alunos da IES pode ser calculada a partir da seguinte fórmula:

Satisfação Geral = -0,095 + 0,377 x F2 (Imagem e Reputação) + 0,243 x F3 (Valor) + 0,218

x F5 (Atendimento aos Alunos) + 0,143 x F1 (Corpo Docente e Currículo)

Portanto, tal como se identificou na análise de correlação, os fatores mais relevantes para a variação da satisfação geral dos alunos são de natureza mais intangível. A imagem e a reputação da IES estão associadas à credibilidade e à confiança nas instituições, nas quais os alunos permanecem geralmente por mais de quatro anos até se graduar. Outro aspecto relevante é o valor, que está relacionado aos benefícios recebidos em função do curso frequentado (neste caso, a Graduação em Administração) e os custos incorridos (valores 
pagos, mensalidades). O atendimento, por sua vez, tem associação com os serviços prestados aos alunos, agregando valor à execução do ensino, o serviço central, representado na análise por meio do corpo docente e do currículo do curso.

\section{CONSIDERAÇÕES FINAIS}

A importância do setor de serviços no contexto social e econômico tem despertado as organizações para a relevância do ato de servir aos clientes, fazendo da prestação de serviços uma fonte de geração de recursos representativa na economia do país. Sendo assim, cada vez mais os prestadores de serviços estão incorporando em suas culturas organizacionais a preocupação com a qualidade dos serviços, demonstrando uma busca constante e persistente pela satisfação de seus clientes.

Em se tratando da competitividade em IESs, a identificação das dimensões (fatores) da qualidade, e seus respectivos atributos, passa a ser uma importante fonte para a consolidação e alavancagem no mercado em que atuam, levando em consideração que alunos satisfeitos têm maior probabilidade de manter relacionamentos e de recomendar a instituição a terceiros. Este estudo se propôs a identificar os atributos e as dimensões da qualidade dos serviços prestados por uma IES com o intuito de direcionar esforços para adequação das necessidades dos clientes (alunos), orientando, assim, a tomada de decisão por parte dos gestores.

Ao final deste estudo, as dimensões da qualidade ou os fatores identificados, em ordem decrescente de percentual de explicação da variância da satisfação geral dos alunos, são: corpo docente e currículo (F1), imagem e reputação da IES (F2), valor (F3), limpeza das instalações (F4), atendimento (F5) e segurança (F6). Os resultados indicaram a relevância do papel do professor na composição das dimensões da qualidade do serviço prestado por IESs, especialmente no que se refere a sua qualificação, experiência e titulação, bem como seu compromisso com o cumprimento do plano de ensino da disciplina ministrada. Além disso, as disciplinas que compõe o currículo do curso, bem como a flexibilidade para cursá-las também se mostram relevantes no que diz respeito à percepção de qualidade do curso de graduação em análise, no caso, o curso de Administração.

A confiança na Instituição e a imagem e reputação que ela possui perante a comunidade também se mostraram aspectos importantes no que se refere à percepção de qualidade por parte dos alunos. Além disso, vale destacar os elementos relacionados ao investimento financeiro do aluno para custear a graduação e à relação entre custo versus 
benefícios (valor para os alunos), observada por eles no decorrer do seu curso de graduação. É interessante observar que a pesquisa foi aplicada em uma IES em que a maioria dos alunos não é contemplada com bolsas de ensino e, portanto, precisam custear seus cursos de graduação. É possível que, em instituições públicas, esta dimensão (ou fator) tenha outra representatividade ou talvez nem componha o rol de fatores relevantes relacionados à qualidade. Pesquisas neste sentido podem ampliar o conhecimento sobre a área e mapear as dimensões em instituições de ensino com outras características.

Ademais, elementos relacionados à manutenção das instalações, especialmente no que se refere à limpeza de corredores, banheiros e salas de aula, também se mostraram relevantes no que se refere à qualidade, constituindo uma dimensão específica. $\mathrm{O}$ atendimento dedicado ao aluno nos departamentos da instituição também se mostra importante, evidenciando a relevância dos diversos momentos da verdade experienciado pelo estudante durante a prestação de um serviço educacional. Isso mostra que, além da relação entre professor e aluno que ocorre na sala de aula, os demais encontros de serviço vivenciados nas instalações de apoio da instituição (como biblioteca e secretaria, por exemplo) também são fatores que merecem atenção da equipe gestora.

Por fim, a segurança na Instituição, seja no interior dos prédios, seja nos acessos e nos estacionamentos, também merece destaque. Neste caso, as equipes que fazem a vigilância dos estabelecimentos de ensino, que, geralmente, são serviços terceirizados, precisam ter sua qualidade ser monitorada constantemente, já que aspectos relacionados à segurança se apresentaram como uma das seis dimensões apresentadas.

Além da identificação das 6 dimensões (fatores) da qualidade dos serviços prestados pela IES, com seus respectivos atributos, foi possível verificar quais destas dimensões mais influenciam na Satisfação Geral dos alunos, sendo elas, a imagem e a reputação, o valor, o atendimento aos alunos e o corpo docente e o currículo do curso. Este resultado se constitui em uma importante informação estratégica, já que emergiu como algo relevante para que as IESs conheçam a origem da satisfação ou da insatisfação de seu público-alvo maior, os alunos, e as maneiras de avaliá-las, para direcionar suas estratégias, ações e investimentos no sentido de qualificarem os serviços prestados e aumentar a satisfação, a retenção e, até mesmo, a lealdade de seus alunos.

Como em toda pesquisa científica, este estudo apresenta limitações, que representam aspectos importantes para a sequência de pesquisas sobre a temática aqui apresentada. A 
primeira limitação que pode ser apontada é o fato da pesquisa quantitativa ter sido realizada por meio de uma coleta de dados do tipo corte transversal único, não permitindo que se verifiquem mudanças na percepção dos respondentes ao longo do tempo. Outra limitação diz respeito sobre a dúvida da existência de outros atributos ou dimensões (fatores) da qualidade dos serviços, que influenciam no momento de decisão por uma determinada IESs ou curso, e que impactam na satisfação geral dos alunos. Assim, outros pesquisadores devem ser encorajados a utilizar o presente estudo como base para outras aplicações em contextos com suas especificidades, as quais podem trazer novas contribuições acadêmicas e gerenciais.

Por fim, outro campo promissor seria a realização da pesquisa com os clientes internos - funcionários e professores - que são os provedores, de fato, dos serviços e o foco de avaliação por parte dos alunos. Este estudo intensificaria a visão interna dos atributos de satisfação e levaria a percepção dos gaps entre o fornecedor (IES) e usuários dos serviços (alunos). O estudo foi aplicado apenas em uma IES e para dar continuidade a um projeto mais amplo de pesquisa, uma vez que se gerou uma base de conhecimento, pode-se aspirar uma validação externa em outras IES, fazendo replicações e melhorias em relação a este estudo.

A pesquisa contempla considerações importantes e de grande valia em relação aos atributos e às dimensões da qualidade que foram identificados, permitindo à IES direcionar planos de trabalho, além de programar melhorias institucionais, com o intuito de oferecer a seus alunos serviços com um padrão diferenciado de qualidade. Sendo assim, o trabalho apresenta contribuições relevantes a respeito dos atributos que compõem as dimensões da qualidade dos serviços que impactam o nível de satisfação geral dos alunos no contexto analisado. Embora sejam contribuições inerentes a um ambiente de pesquisa único, com suas especificidades, há a possibilidade de se ampliar a discussão e de se replicar pesquisas similares em outros contextos, gerando melhorias na qualidade dos cursos de graduação em Administração no Brasil, aumentando o nível de satisfação dos alunos e estimulando a sua retenção junto às IES. Para se construir uma percepção de qualidade elevada dos alunos, cabe aos gestores das IESs avaliar cuidadosamente cada uma das dimensões (fatores) e projetar estratégias e ações potencializem a satisfação geral de seus alunos.

\section{REFERÊNCIAS}

ALVES, H.; RAPOSO, M. Conceptual model of student satisfaction in higher education. Total Quality Management. v. 18, n. 5, p. 571-588, 2007. 
ANDERSON, E. W.; FORNELL, C.; LEHMANN, D. Customer satisfaction, market share and profitability: findings from Sweden. Journal of Marketing, v. 58, n. 3, p. 53-66, 1994.

BERGAMO, F. V. M.; GIULIANI, A. C.; GALLI, L. C. L. A. Modelo de lealdade e retenção de alunos para instituições do ensino superior: um estudo teórico com base no marketing de relacionamento. Brazilian Business Review, v. 8, n. 2, p. 43-67, 2011.

BERRY, L. L. Descobrindo a essência do serviço: os nove geradores de sucesso sustentável nos negócios. Rio de Janeiro: Qualitymark, 2001.

DABHOLKAR, P. A. A contingency framework for predicting causality between customer satisfaction and service quality. Advances in Consumer Research, v. 22, n. 1, p. 101-108, 1995.

DAVEY, A.; SAVLA J. Statistical power analysis with missing data. London: Routledge, 2009.

DENZIN, N. K.; LINCOLN, Y. S. The Sage handbook of qualitative research. $4^{\text {th }}$ edition. Thousand Oaks: Sage Publications, 2011.

EBERLE, L.; MILAN, G. S.; LAZZARI, F. Identificação das dimensões da qualidade em serviços: um estudo aplicado em uma instituição de ensino superior. RAE-eletrônica, v. 9, n. 2, p. 1-32, 2010.

ELLIOT, K. M.; SHIN, D. Student satisfaction: an alternative approach to assessing this important concept. Journal of Higher Education Policy and Management, v. 24, n. 2, p. 197-209, 2002.

ENDERS, C. K. Applied missing data analysis. New York: The Gilford Press, 2010. FERREIRA, A. Uma análise das dimensões da qualidade de serviços em educação superior para o estabelecimento de cursos de pós-graduação. Dissertação (Mestrado Profissionalizante em Engenharia de Produção). Porto Alegre: Universidade Federal do Rio Grande do Sul, 2005.

FINK, A. How to conduct surveys: a step-by-step guide. $5^{\text {th }}$ edition. Thousand Oaks: Sage Publications, 2012.

FORNELL, C. A national customer satisfaction barometer: the Swedish experience. Journal of Marketing, v. 56, n. 1, p. 6-21, 1992.

GREY, C. Reinventing business schools: the contribution of critical management education. Academy of Management Learning and Education, v. 3, n. 2, p. 178-186, 2004.

GUBRIUM, J. F.; HOLSTEIN, J. A.; MARVASTI, A. B.; MCKINNEY, K. D. The Sage handbook of interview research: the complexity of the craft. $2^{\text {nd }}$ edition. Thousand Oaks: Sage Publications, 2012. 
HAIR Jr., J. F.; ANDERSON, R. E.; TATHAM, R. L.; BLACK, W. C. Análise multivariada de dados. 6. ed. Porto Alegre: Bookman, 2009.

HAIR Jr., J. F.; WOLFINBARGER, M.; ORTINAU, D. J.; BUSH, R. P. Fundamentos de pesquisa de marketing. Porto Alegre: Bookman, 2010.

JACKSON, M. J.; HELMS, M. M.; AHMADI, M. Quality as a gap analysis of college students expectation. Quality Assurance in Education, v. 19, n. 4, p. 392-412, 2011.

JOHNSON, R. A.; WICKERN, D. W. Applied multivariate statistical analysis. $6^{\text {th }}$ edition. Upper Saddle River: Pearson / Prentice Hall, 2007.

JONGBLOED, B. Marketization in higher education: Clark's triangle and the essential ingredients of markets. Higher Education Quarterly, v. 57, n. 2, p. 110-135, 2003.

KANJI, G. K. Measuring business excellence. London: Taylor and Francis Group, 2007.

KING, N.; HORROCKS, C. Interviews in qualitative research. Thousand Oaks: Sage Publications, 2010.

KLINE, R. B. Principles and practice of structural equation modeling. $3^{\text {rd }}$ edition. New York: The Gilford Press, 2011.

KOTLER, P.; FOX, K. F. A. Marketing estratégico para instituições educacionais. São Paulo: Atlas, 1994.

KRIPPENDORFF, K. Content analysis: an introduction to its methodology. $3^{\text {rd }}$ edition. Thousand Oaks: Sage Publications, 2012.

LAROCHE, M.; KALAMAS, M; CHEIKHROUHOU, S.; CÉZARD, A. An assessment of the dimensionality of should and will service expectations. Canadian Journal of Administrative Sciences, v. 21, n. 4, p. 361-375, 2004.

LEECH, N. L.; BARRET, K. C.; MORGAN, G. A. SPSS for intermediate statistics: use and interpretation. $2^{\text {nd }}$ edition. New Jersey: Lawrence Erlbaum Associates, 2005.

LOPES, H. E. G.; LEITE, R. S.; LEITE, D. S. O que realmente importa? Um estudo sobre os fatores determinantes da qualidade percebida no curso superior de uma instituição do CentroOeste de Minas Gerais. Revista Eletrônica de Administração, v. 13, n. 2, 2007.

LOVELOCK, C. H. Classifying services to gain strategic marketing insights. Journal of Marketing, v. 47, n. 3, p. 9-20, 1983.

LOVELOCK, C. H.; WRIGHT, L. Serviços: marketing e gestão. São Paulo: Saraiva, 2001.

LOURENÇO, C. D. S.; KNOP, M. F. T. Ensino superior em administração e percepção da qualidade de serviços: uma aplicação da escala SERVQUAL. Revista Brasileira de Gestão de Negócios, v. 13, n. 39, p. 219-233, 2011. 
MAINARDES, E. W.; DOMINGUES, M. J. C. S. Avaliação da qualidade nos serviços educacionais das instituições de ensino superior em Joinville, SC. Revista de Gestão (USP), v. 16, n. 1, p. 17-32, 2009.

MALHOTRA, N. K. Marketing research: an applied orientation. $5^{\text {th }}$ edition. New Jersey: Prentice Hall, 2006.

MEYER Jr., LOPES, M. C. Planejamento formal e seus resultados: um estudo de caso de universidades. In: Seminários de Pesquisa em Educação da Região Sul. 5. Anais... Curitiba, 2004.

MEYERS, L. S.; GAMST, G. C.; GUARINO, A. J. Applied multivariate research: design and interpretation. Thousand Oaks: Sage Publications, 2012.

MILAN, G. S.; BRENTANO, J.; DE TONI, D. A qualidade percebida dos serviços prestados por uma agência de comunicação e satisfação do cliente: um estudo exploratório. Revista Brasileira de Gestão de Negócios, v. 10, n. 26, p. 17-26, 2008.

MILAN, G. S.; DE TONI, D.; MAIOLI, F. C. Atributos e dimensões relacionadas aos serviços prestados por uma instituição de ensino superior e a satisfação de alunos. Gestão e Planejamento, v. 13, n. 2, p. 199-214, 2013.

MILES, M. B.; HUBERMAN, A. M.; SALDAÑA, J. Qualitative data analysis: a methods sourcebook. $3^{\text {rd }}$ edition. Thousand Oaks: Sage Publications, 2013.

MORALES, M.; CALDERÓN, L. F. Assessing service quality in schools of business: dimensions of service in continuing professional education (CPE). In: BALAS - Latin America's New Millennium. Proceedings..., p. 524-536, 1999.

MULAIK, S. A. Foundations of factor analysis. $2^{\text {nd }}$ edition. Boca Raton: Taylor \& Francis Group, 2010.

OLIVER, R. L. Satisfaction: a behavioral perspective on the consumer. $2^{\text {nd }}$ edition. New York: The McGraw-Hill, 2010.

O'NEILL, M. A.; PALMER, A. Importance-performance analysis: a useful tool for directing continuous quality improvement in higher education. Quality Assurance in Education, v. 12 , n 1, p. 39-52, 2004.

OSTROM, A.; IACOBUCCI, D. Consumer trade-offs and the evaluation of services. Journal of Marketing, v. 59, n. 1, p. 17-28, 1995.

PANTOUVAKIS, A.; LYMPEROPOULOS, K. Customer satisfaction and loyalty in the eyes of new and repeat customers: evidence from the transport sector. Managing Service Quality, v. 18, n. 6, p. 623-643, 2008. 
PARASURAMAN, A.; ZEITHAML, V. A.; BERRY, L. L. A conceptual model of service quality and its implications for future research. Journal of Marketing, v. 49, n. 4, p. 41-50, 1985.

PARASURAMAN, A.; ZEITHAML, V. A.; BERRY, L. L. SERVQUAL: a multiple-item scale for measuring consumer perception of service quality. Journal of Retailing, v. 64, n. 1, p. 12-40, 1988.

REED, J.; VAKOLA, M. What role can a training needs analysis play in organizational change? Journal of Organisational Change Management, v. 19, n. 3, p. 393-407, 2006.

REICHHELD, F. F.; MARKEY Jr.; R. G.; HOPTON, C. The loyalty effect: the relationship between loyalty and profits. European Business Journal, v. 12, n. 3, p. 134-139, 2000.

REMLER, D. K.; VAN RYZIN, G. G. Research methods in practice: strategies for description and causation. Thousand Oaks: Sage Publications, 2011.

RIBEIRO, J. L. D.; MILAN, G. S. Planejando e conduzindo entrevistas individuais. In: RIBEIRO, J. L. D.; MILAN, G. S. (eds.) Entrevistas individuais: teoria e aplicações. Porto Alegre: FEENG/UFGRS, 2004. cap. 1, 9-22.

SULTAN, P.; WONG, H. Y. Antecedents and consequences of service quality in a higher education context: a qualitative research approach. Quality Assurance in Education, v. 21, n. 1, p. 70-95, 2013.

TABACHNICK, B. G.; FIDELL, L. S. Using multivariate statistics. $6^{\text {th }}$ edition. Boston: Allyn and Bacon, 2012.

XAVIER, A. C. R. A gestão da qualidade e a excelência dos serviços educacionais: custos e benefícios de sua implantação. Brasília: IPEA, 1996.

ZEITHAML, V. A. Service quality, profitability, and the economic worth of customers: what we know and what we need to learn. Journal of the Academy of Marketing Science, v. 28, n. 1, p. 67-85, 2000.

ZEITHAML, V. A.; BITNER, M. Jo. Marketing de serviços: a empresa com foco no cliente. Porto Alegre: Bookman, 2003. 